\title{
In vitro differentiation of bone marrow derived porcine mesenchymal stem cells into endothelial cells
}

\author{
Divya Pankajakshan, Vikash Kansal, and Devendra K. Agrawal ${ }^{\star}$ \\ Centre for Clinical and Translational Science, Creighton University School of Medicine, Omaha, \\ NE, USA
}

\section{Abstract}

\begin{abstract}
Mesenchymal stem cells (MSCs) hold potential for the regeneration of damaged tissues in cardiovascular diseases. In this study, we investigated the potential of porcine MSCs to differentiate into endothelial cells (ECs) in-vitro. The cultured bone marrow derived cells were $\mathrm{CD} 11 \mathrm{~b}^{-} \mathrm{CD} 34^{-} \mathrm{CD} 44^{+} \mathrm{CD} 45^{-} \mathrm{CD} 90^{+}$and showed mesodermal lineage differentiation which are characteristic of MSCs. The MSCs were induced to differentiate into ECs using endothelial growth media (EGM) with and without high concentrations of VEGF (EGM+VEGF; 50ng/ml). The endothelial basal medium (EBM) without growth factors served as the control. The EC differentiation was assessed by the presence of $\mathrm{vWF}$, ability to take up acetylated LDL, in vitro angiogenesis assay, flow cytometry and qPCR of EC markers vWF, VE-cadherin, PECAM-1, CD34, VEGF-R1 and VEGF-R2 after 10days of stimulation. The cells cultured in EGM+VEGF media demonstrated higher amount of DiI-AcLDL positive cells, and enhanced presence of vWF (90\%), VE-Cadherin (60\%) and PECAM-1 (48\%) positive cells, than in EBM. These cells showed profuse sprouting of capillary tubes and closed polygon formation in the angiogenesis assay. There was 1.5-2 fold increase in the mRNA expression of endothelial markers in the cells stimulated with EGM+VEGF media when compared to control. The results demonstrate the ability of porcine MSCs to differentiate into ECs under in-vitro inducing conditions. The differentiated cells would provide new options for re-endothelialization following interventional procedures and tissue engineering.
\end{abstract}

\section{Introduction}

\begin{abstract}
Mesenchymal stem cells (MSCs) offer remarkable potential in regenerative medicine, and have been investigated for the treatment of vascular diseases. They are present in various organs, including bone marrow, adipose tissue (Zuk et al., 2001), umbilical cord blood (Lee et al., 2004), liver and spleen (Kuo et al., 2008). Bone marrow is one of the most widely studied sources for MSCs mainly because of the ease and accessibility of MSCs through aspiration procedures compared to other sources (Peister et al., 2004). MSCs represent
\end{abstract}

\footnotetext{
"Corresponding author: Devendra K. Agrawal, M.Sc. (Chem), Ph.D. (Biochem), Ph.D. (Med. Sciences), MBA, MS (ITM), FAAAAI, FAHA, Associate Dean for Translational Research, Director, Center for Clinical \& Translational Science, The Peekie Nash Carpenter Endowed Chair in Medicine, Professor of Biomedical Sciences, Internal Medicine, and Medical Microbiology \& Immunology, Creighton University School of Medicine, CRISS II Room 510, 2500 California Plaza, Omaha, NE 68178, USA, Tel: 402-280-2938; Fax: 402-280-1421, dkagr@creighton.edu.

Author disclosure statement

No competing financial interests exist.
} 
approximately $0.0001 \%$ of nucleated bone marrow cell population (Pittenger et al., 1999). They are characterized as plastic adherent population, expressing CD105, CD73 and CD90, and lacking the expression of CD45, CD34, CD11b, CD19 and HLA-DR surface molecules, and have multilineage differentiation potentials (Dominici et al., 2006). They can be differentiated into cells of mesenchymal lineage, including osteocytes, chondrocytes, adipocytes and hematopoiesis supporting stromal cells (Prockop, 1997). MSCs have also been reported, albeit controversially, to differentiate into myocytes (Wakitani et al., 1995) and cardiomyocytes (Toma et al., 2002) and even into cells of non-mesodermal origin, including hepatocytes and neurons (Jiang et al., 2002). The potent immunosuppressive functions of MSCs have been demonstrated both in vitro and in vivo (Ghannam et al., 2010).

Strategies to generate differentiated vascular cells from MSCs will enhance potential use of autologous vascular progenitor cells for therapeutic transplantation as well as in tissue engineering. In mammals, vascular progenitors first appear in the yolk sac, where mesodermal precursors of both hematopoietic and endothelial lineages differentiate into solid clumps known as blood islands. The outer cells of these blood islands become flattened and differentiate into ECs, while the inner cells become hematopoietic cells (De Val and Black, 2009). Several populations of cells derived from the bone marrow have a potential to differentiate into endothelial cells (ECs). The endothelial progenitor cells (EPCs) that originate in the bone marrow play a role in vasculogenesis (physiological and pathological) and circulate in adult peripheral blood (Asahara et al., 1997). Multipotent adult progenitor cell (MAPC) which are CD34-, vascular endothelial cadherin ${ }^{-}$(VE-cadherin ${ }^{-}$), $\mathrm{AC}^{-} 33^{+}$, and fetal liver kinase ${ }^{+}\left(\mathrm{Flk1}^{+}\right)$, when cultured with VEGF differentiated into CD34+, VEcadherin ${ }^{+}$, Flk $1^{+}$cells, a phenotype that would be expected for angioblasts (Reyes et al., 2002). The MAPCs subsequently differentiate into cells that express endothelial markers, function in vitro as mature ECs, and contributed to neo-angiogenesis in vivo during tumor angiogenesis and wound healing. In an interesting work, Salven et al. (2003) demonstrated that $\mathrm{CD} 133^{+} \mathrm{CD} 34^{+}$cells co-expressing VEGFR-3 are functionally non-adherent EC precursors that have the capacity to differentiate into mature adherent VEGFR- $3^{+}$EC. Although MSCs can differentiate into various cell types, the endothelial commitment of porcine mesenchymal stem cells is less investigated.

The aim of this study was to evaluate the differentiation of porcine MSCs into ECs in in vitro culture for potential in vivo application in cardiovascular disease therapy and tissue engineering. Porcine species were selected since they are considered to be a valuable system for preclinical assessments in cardiovascular diseases due to their anatomical and physiological similarities with humans. In this study, we first characterized the porcine bone marrow derived cells and further showed their ability for EC differentiation through functional assays, and mRNA and protein expression analysis of EC markers.

\section{Materials and Methods}

\subsection{Isolation and culture of porcine MSCs}

Tibias and femurs from freshly sacrificed 3-5 month old pigs were obtained from the slaughterhouse in sterile Hanks balanced salt solution (HBSS) containing antibiotics. MSCs were isolated by combining Ficoll gradient centrifugation and plastic adherence technique. 
Briefly, the bone marrow cell suspension after dilution with equal volume of HBSS and was layered over Histopaque 1077 and the tubes were centrifuged at $400 \mathrm{~g}$ for $30 \mathrm{~min}$ at room temperature. Mononuclear cells recovered from the distinct layer at the plasma-Histopaque interface were washed twice with Dulbecco's Modified Eagle's Medium-Knock out medium (DMEM-KO; GibcoBRL, Grand Island, NY, USA) by centrifugation ( $350 \mathrm{~g}, 5 \mathrm{~min}$ ) to remove any remaining Histopaque. After the final centrifugation, the supernatant was discarded and the cell pellet was resuspended in complete medium containing $5 \mathrm{ml}$ of DMEM-KO containing 10\% MSC specific fetal bovine serum (FBS) (GibcoBRL, Grand Island, NY, USA), 2mM Glutamax (Invitrogen, Carlsbad, CA, USA) and 1\% penicillinstreptomycin solution (Sigma, St. Louis, MO, USA). Medium was changed twice per week. Cell concentration was determined using a coulter counter (Beckman coulter, Brea, CA, USA) and cells were plated at a density of $4 \times 10^{5} / \mathrm{cm}^{2}$. The media was replaced after $24 \mathrm{~h}$ and then every 3-4 days afterward. Cultures reached confluency after 8-10 days and were passaged by trypsinization. The cultures were maintained at $37^{\circ} \mathrm{C}$ in a humidified atmosphere containing $5 \% \mathrm{CO}_{2}$ in complete medium. Cells in the passage 3 to passage 5 were used for characterization experiments.

\subsection{Characterization of MSCs}

2.2.1. Immunophenotyping-Flow cytometry analysis was done for identifying Macrophage marker CD11b, hematopoietic stem cell markers CD34, CD45 and MSC markers CD44 and CD90. Cells $\left(1 \times 10^{6} / \mathrm{ml}\right)$ were washed with PBS containing 4\% FBS and incubated with monoclonal antibodies, Antihuman CD11b-APC (Clone: ICRF44; ebiosciences, San Diego, CA, USA), Antihuman CD34-FITC (Clone: 581; BD Pharmingen, Franklin Lakes, NJ, USA), Antihuman CD45-FITC (Clone: 2D1; ebiosciences), Antihuman CD44-FITC (Clone: IM7; ebiosciences), Antihuman CD90-PE (Clone: 5E10; ebiosciences) for $30 \mathrm{~min}$ at $4{ }^{\circ} \mathrm{C}$ in dark. The dilution and concentration of antibodies used were as specified by the manufacturer. The cells were further washed 3 times in PBS and resuspended in 500 $\mu \mathrm{PBS}$ and flow cytometry was performed on a FACS Aria Flow Cytometry System (BD Biosciences, San Jose, CA, USA). FITC, APC and PE-labeled IgG (BD Pharmingen, Franklin Lakes, NJ, USA) served as the isotype control.

2.2.2. Immunostaining for CD105 and Vimentin-For vimentin staining, the cells were fixed with $3.7 \%$ formaldehyde in PBS for $100 \mathrm{~min}$. The fixed monolayer was rinsed with PBS three times and permeabilized by incubating with $0.1 \%$ Triton-X 100 in PBS for 10 min. After PBS wash, the cells were blocked in 1\% BSA in PBS for 1 hour and was incubated with Vimentin antibody (1:100 dilution; sc-73259, SantaCruz biotechnology, SantaCruz, CA, USA) for $1 \mathrm{~h}$ at RT. The cells were further washed with PBS and incubated with the secondary antibody for $30 \mathrm{~min}$. The cells were further washed with PBS and were mounted in Vectashield with DAPI (Vector Laboratories, Burlingame, CA, USA). For CD105 staining, the cells were directly stained using CD105-PE (eBiosciences, San Diego, CA, USA) after the fixation, washing and blocking steps. After PBS wash, the cells were mounted in Vectashield mount containing DAPI and viewed using a fluorescence microscope (Olympus BX51, St. Louis, MO, USA). 
2.2.3. Differentiation assays-The MSCs were stimulated using STEMPRO osteogenesis, chondrogenesis and adipogenesis differentiation kits (Gibco BRL, Grand Island, NY, USA) for osteocyte, chondrocyte and adipocyte differentiation, respectively. For osteogenic and adipogenic differentiation, the cells were cultured on chamber slides and the induction medium was added at $80 \%$ confluency. The cells were analyzed for osteogenesis by Alizarin S Red staining after 14 days and for adipogenic differentiation by Oil O Red staining after 21 days of stimulation. For chondrogenic differentiation the cell pellet was cultured for 21 days in the inducing medium and was snap frozen. Cryosections of $5 \mu \mathrm{m}$ thickness were taken stained by Safranin O and Alcian blue and was observed under a bright field microscope. All the histochemical reagents were obtained from Sigma (St. Louis, MO, USA).

2.2.4. RNA Isolation, Reverse Transcription and RT-PCR-Total RNA was isolated from MSC at $2 \mathrm{P}$ to $4 \mathrm{P}$ for analysis of MSC marker expression. Real time PCR analysis was done for the identification of CD11b, CD73, CD90, CD105 and CD106 mRNA transcripts. The total RNA was isolated using the Trizol reagent (Sigma, St. Louis, MO, USA) method. The yield of RNA was quantified using Nanodrop (Thermo scientific, Rockford, IL, USA). First-strand cDNA synthesis was done using $1 \mu \mathrm{g}$ total RNA with oligo dT $(1 \mu \mathrm{g}), 5 \mathrm{X}$ reaction buffer, $\mathrm{MgCl}_{2}$, dNTP mix, RNAse inhibitor and Improm II reverse transcriptase as per Improm II reverse transcription kit (Promega, Madison, WI, USA). Following the first strand synthesis, Real time PCR was done using $8 \mu \mathrm{l}$ cDNA, $10 \mu \mathrm{l}$ SYBR green PCR master mix (BioRad Laboratories, Hercules, CA, USA) and forward and reverse primers (10 picomol/ $\mu$ l) (Integrated DNA Technologies, San Diego, CA, USA) using a Real Time PCR system (CFX96, BioRad Laboratories, Hercules, CA, USA). The specificity of the primers was analyzed by running a melting curve. The PCR cycling conditions used were $5 \mathrm{~min}$ at $95{ }^{\circ} \mathrm{C}$ for initial denaturation, 40 cycles of $30 \mathrm{~s}$ at $95^{\circ} \mathrm{C}, 30 \mathrm{~s}$ at $52-58^{\circ} \mathrm{C}$ (depending upon the primer annealing temperatures) and $30 \mathrm{sec}$ at $72^{\circ} \mathrm{C}$. Each real-time PCR was carried out using 4 individual samples in duplicates and the threshold cycle values were averaged. Calculations of relative normalized gene expression were done using the Biorad CFX manager software based on the ${ }^{\Delta \Delta} \mathrm{Ct}$ method. The results were normalized against housekeeping gene glyceraldehyde-3-phosphate dehydrogenase (GAPDH). Statistical analysis of real-time quantitative PCR results was expressed using non-parametric MannWhitney $\mathrm{U}$ test to evaluate significant differences between groups.

\subsection{EC characterization of differentiating MSCs}

2.3.1. Media composition for EC differentiation-MSCs in 3P-5P were used for EC differentiation studies. The stimulation was started when the cells were $60-70 \%$ confluent. The MSC culture was stimulated with three different types of medium; Endothelial basal medium containing 2\% FBS (EBM) and no added growth supplements, Endothelial basal medium containing growth supplements and 2\%FBS (EGM) (EGM-2 bullet kit, Lonza, Walkersville, MD, USA) and finally the EGM+VEGF medium which is EGM medium containing increased concentrations of VEGF165 (50ng/ml) (Peprotech, Rocky Hill, NJ, USA). The growth supplements added in the EGM medium are hydrocortisone, human fibroblast growth factor (hFGF- $\beta$ ), VEGF, R3-IGF-1, ascorbic acid, human epithelial growth factor (hEGF), GA-1000, and heparin (EGM-2 bullet kit; Lonza, Walkersville, MD, USA) 
(amounts are proprietary and undisclosed). The EC differentiation was analyzed after 10days of stimulation. Medium was replaced every 3 days. The cells were maintained in a $\mathrm{CO} 2$ incubator at $37^{\circ} \mathrm{C}$ and $5 \% \mathrm{CO} 2$.

2.3.2. Immunostaining for vWF-MSCs after stimulation with the induction medium for 10days were fixed using 3.7\% formaldehyde in PBS, washed with PBS, quenched for 20 min with $0.27 \% \mathrm{NH} 4 \mathrm{Cl} / 0.38 \%$ glycine in PBS and was permeabilized with $0.1 \%$ Triton$\mathrm{X} 100$ in PBS for 10min. After washing with PBS, the cells were blocked with $1 \%$ BSA in PBS for $30 \mathrm{~min}$. The cells were further incubated with vWF antibody (sc-73268, SantaCruz biotechnology, SantaCruz, CA, USA) for $1 \mathrm{~h}$ at RT. After 3 PBS washes, the cells were incubated with the secondary antibody (FITC-goat antimouse IgG, F2761, Invitrogen, Molecular Probes, Carlsbad, CA, USA) for $30 \mathrm{~min}$. After washing in PBS, the cells were mounted using Vectashield mounting medium (Vector Laboratories, Burlingame, CA) and was observed using an upright fluorescent microscope (Olympus BX51, St. Louis, MO, USA).

2.3.3. Dil-Acetylated LDL uptake - The stimulated MSCs in culture were washed twice with serum free medium and then incubated with $10 \mu \mathrm{g} / \mathrm{ml}$ DiI-acetylated LDL (Molecular Probes, Carlsbad, CA) in serum free medium for $4 \mathrm{~h}$ at $37^{\circ} \mathrm{C}$ and $5 \% \mathrm{CO}_{2}$. The cell layer was washed in PBS and was observed using an inverted fluorescent microscope (Olympus CKX41, St. Louis, MO).

2.3.4. Immunophenotyping-After 10days stimulation the cells were trypsinized and flow cytometry analysis was done for identifying EC markers vWF, PECAM-1 and VECadherin. Cells were aliquoted to a concentration of $1 \times 10^{6}$ cells per tube, fixed using $0.2 \%$ formaldehyde permeabilized using 0.1\% Triton-X 100 in PBS and were individually incubated with monoclonal antibodies, for vWF, PECAM-1 and VE-Cadherin (1:100 dilution; Santacruz biotechnology) for $45 \mathrm{~min}$. The cells were then washed three times in PBS and further incubated with the secondary antibody conjugated to FITC for $30 \mathrm{~min}$ (1:1000 dilution; Invitrogen, Carlsbad, CA). The cells were further washed 3 times in PBS and resuspended in 500 $\mu$ PBS and flow cytometry was performed on a FACS Aria Flow Cytometry System (BD Biosciences, San Jose, CA). The cells stained with the secondary antibody alone served as control.

2.3.5. Angiogenesis assay-The MSCs after stimulation for 1odays using the defined medium composition were harvested and angiogenesis assay was done according to manufacturer's protocol (Chemicon, Temecula, CA). Polymerized EC matrices were prepared by incubating the $100 \mu \mathrm{l}$ of ECM gel matrix solution into each well of a 24 -well plate, at $37^{\circ} \mathrm{C}$ for $1 \mathrm{~h}$. The stimulated cells were seeded at a concentration of $1 \times 10^{4}$ cells on EC matrices. The EGM media $(300 \mu \mathrm{l})$ was added into each well and was maintained at $37^{\circ} \mathrm{C}$ and $5 \% \mathrm{CO}_{2}$ in a $\mathrm{CO}_{2}$ incubator for $12 \mathrm{~h}$. The formation of capillary tubes was analyzed using an inverted phase contrast microscope (Olympus CKX41, St. Louis, MO).

2.3.6. RNA Isolation, Reverse Transcription and Real Time PCR-The total RNA was isolated after stimulation for 10days with defined medium and reverse transcription and Real-time PCR was done as stated before. The primer sequences used for vWF, VE- 
Cadherin and PECAM-1 are given in Table 1. The results were normalized against glyceraldehyde-3-phosphate dehydrogenase (GAPDH). Statistical analysis of real-time quantitative PCR results was expressed using non-parametric Mann-Whitney $U$ test to evaluate significant differences between groups.

\section{Results and discussion}

MSCs are non-hematopoietic progenitor cells isolated from adult tissues. The ease of isolation, rapid growth in vitro allowing for extensive culture expansion, maintenance of differentiation potential, immune-modulatory effects, and their ability to home to the sites of injury and subsequent wound healing, makes MSCs attractive candidates for regenerative medicine. In this study, we isolated and characterized porcine bone marrow derived MSCs and demonstrated the potency to these cells to differentiate into ECs in vitro. Mesenchymal stem cells from porcine species have not been well characterized in general. Hence we thoroughly characterized the MSCs and further demonstrated its differentiation.

\subsection{MSC characterization}

3.1.1. Immunophenotyping-After the initial cell seeding, the medium was removed every $24 \mathrm{~h}$ till $72 \mathrm{~h}$ to remove the hematopoietic cells. The primary culture was established after 7days of culture. The adherent cells showed fibroblastoid morphology (Fig 1. iA) and stained positively for vimentin (Fig 1. iB) and CD105 (Endoglin) (Fig 1. iC) that are characteristic for MSCs. Flow cytometry data revealed that the cells at the second and fourth passages, stained negative for CD11b (macrophage marker) (Fig 1. ii A\&F), CD34 and CD45 (hematopoietic stem cell markers) (Fig 1. ii B\&D, G\&I), showing negligible contamination by macrophages and hematopoietic stem cells in culture. The cells expressed CD44 (Fig 1. iiC\&H) and CD90 (Fig 1. iiE\&J), indicating that their origin from a mesenchymal lineage. The frequent change of medium after seeding was effective in preventing the attachment of hematopoietic stem cells and other non-adherent cells.

3.1.2. Real Time PCR-Real time PCR revealed mRNA expression of MSC markers, CD73, CD90, CD105 and CD106 from 2P to 6P (Fig 2. i). There was no significant change in the expression of these markers when the cells from different passages were compared. There was no detectable CD11b mRNA expression in the cells of any of these passages. The results confirmed mRNA expression of markers CD73, CD90, CD105 and CD106, which confirmed the mesenchymal origin of the cells. The expressions of these markers were consistent as we analyzed from $2 \mathrm{P}$ to $6 \mathrm{P}$. This showed that the surface expression of these markers was not altered in culture and that the cells were not trans-differentiating.

3.1.3. Multilineage differentiation potential of MSCs-The multilineage potential of the isolated cells was proved by differentiation of the isolated cells into osteogenic, chondrogenic and adipogenic lineages. The cells after 14 days stimulation with osteogenic differentiation medium showed presence of calcium deposits, as revealed by Alizarin $\mathrm{S}$ red as orange red stain (Fig 2. iiA\&B). The adipogenic differentiation of cells after 21 days stimulation was confirmed by the presence of lipid droplets within the cells by Oil O Red staining (Fig 2. iiC). The cryosections of the cell pellet, after 21days of stimulation with chondrogenic differentiation medium (Fig 2. iiD) stained orange-green for cartilage by 
Safranin O (Fig 2. iiE) and stained blue for the glycosaminoglycans by Alcian blue (Fig 2 . iiF), confirming the differentiation of the cells into cartilage. The cells showed differentiation to osteocytes, chondrocytes and adipocytes, which confirmed the multipotentiality to differentiate to mesodermal lineages. The phenotype, the expression of surface markers and the multilineage differentiation capability provided convincing evidence that the cells isolated in the present study were indeed MSCs.

\subsection{Endothelial cell differentiation}

Previous reports demonstrated in vitro EC differentiation from various stem cell sources such as EPCs, embryonic stem cells (ESC) and MAPCs. Nourse and colleagues (2010) reported that VEGF treatment for 14days at $50 \mathrm{ng} / \mathrm{ml}$ resulted in a 4.7 -fold increase in the number of ESCs differentiating to ECs. They observed increased expression of CD31, VECadherin, and vWF with an increase in the VEGF dosage which reached a plateau beyond 50ng/ml. McCloskey and colleagues (McCloskey et al., 2003) isolated highly enriched populations of actively proliferating EC populations from murine embryonic stem cell line, when the selected flk- $1^{+}$outgrowth cells were stimulated with EGM containing VEGF and expanded on collagen IV matrix. In another study, human ESCs on stimulation with EGM media showed increased levels of EC markers PECAM1, VE-Cadherin, and CD34 reaching a maximum at days 13-15 and indicating a differentiation process towards ECs (Levenberg et al., 2002). Kaufman and colleagues (Kaufman et al., 2004) differentiated rhesus monkey ESCs to endothelial-like cells using EGM media after a stimulation of 29days. The cells expressed vWF, Flk-1 and demonstrated Ac-LDL uptake. However, the cells did not express VE-Cadherin and PECAM-1. MAPCs cultured in serum free medium with 20ng/ml VEGF165 showed significantly increased mRNA expression of EC markers vWF, VECadherin, CD31, eNOS and Flk-1 by day 10 (Xu et al., 2008). However, to our knowledge there are no well-documented studies reported on the differentiation of porcine mesenchymal stem cells into endothelial cells.

A panel of antigenic and functional assays was done for the characterization of ECs differentiated from MSCs. These include: von Willebrand factor, a multimeric glycoprotein, synthesized in ECs and stored in the Weibel-Palade bodies (Mannucci and Federici, 1995); CD31 (PECAM-1), a glycoprotein expressed by ECs at the endothelial cell junction, and VE-cadherin, which is specifically localized to the inter-endothelial cell junction (Lampugnani et al., 1992). All three EC markers were expressed after the induction.

3.2.1. Immunostaining-After 10days stimulation with EBM media there was no significant change in the cell phenotype (Fig 3A). But, there was a marked difference in the cell phenotype after differentiation in EGM and EGM+VEGF media (Fig 3B,C). The cells appeared to show a cobble stone morphology that is characteristic of ECs.

Lipoproteins labeled with the fluorescent probe, DiI, which metabolically label ECs, have been utilized to visualize the uptake of lipoproteins thus facilitating their identification by fluorescence microscopy (Voyta et al., 1984). In the current study, the cells stimulated with EGM (Fig 3E) and EGM+VEGF (Fig 3F) media showed significant increase in the acetylated LDL uptake which may be attributed to the MSC differentiation into ECs. The 
EGM +VEGF stimulated cells showed increased percentage of DiI Ac $\mathrm{LDL}^{+}$cells compared to that of EGM. The cells stimulated with the EBM medium was entirely negative for DiI Ac LDL (Fig 3D). The characteristic staining of vWF could be visualized in significantly higher number of cells cultured in the EGM (Fig 3H) and EGM+VEGF (Fig 3I) which is localized in the Weibel Palade bodies of the cells and are distributed throughout the cytoplasm. There was no expression of vWF in the cells cultured in the control EBM medium (Fig 3G).

3.2.2. Angiogenesis assay-The cells after 10days stimulation were seeded onto the ECM gel. The cultured cells in EBM-2 media showed formation of few capillary tubes, but there was no closed polygon formation and the sprouting of new capillary tubes (Fig 4A). In the cells cultured in EGM media, the sprouting of new capillary tubes and formation of closed polygons were clearly visible (Fig 4B). There was development of complex mesh like structures in addition to closed polygon formation in cells cultured in EGM + VEGF media. The sprouting of new capillary tubes was profuse when compared to that of EGM media (Fig 4C). The profuse capillary formation in the cells induced with EGM + VEGF media demonstrates a significant role of the concentration of VEGF in the media in the differentiation of MSCs to ECs.

3.2.3. EC marker expression by differentiating MSCs-The cells cultured in the EBM medium did not show expression of EC markers (Fig. 5A, D\&G). After 10 days exposure in EGM medium the cells showed a significantly higher number of cells with positive expression of vWF $(87 \pm 3 \%$ ) (Fig. 5B), VE-Cadherin (24 $\pm 5 \%$ ) (Fig. 5E), and PECAM-1(18 $\pm 3 \%$ ) (Fig. 5H). The increased concentrations of VEGF in EGM resulted in remarkably enhanced expression of vWF+ $(90 \pm 4 \%)$ (Fig. 5C), VE-Cadherin $(60 \pm 5 \%)$ (Fig. $5 \mathrm{~F}$ ), and PECAM-1(48 $\pm 4 \%$ ) (Fig 5I). There was no significant difference in the vWF expression in the cells stimulated with EGM $(87 \pm 3 \%)$ and EGM+VEGF $(90 \pm 4 \%)$.

The higher percentage of vWF expression in the differentiating cells in EGM and EGM +VEGF media, when compared to the other markers, VE-Cadherin and PECAM-1, might be due to the fact that $\mathrm{VWF}$ is expression is initiated even in the premature ECs, whereas VECadherin and PECAM-1 are expressed in mature ECs. Ostwald et al. (2004) have reported the differentiation of ECs from human bone marrow derived MSCs. In their study, the MSCs acquired major characteristics of mature endothelial-like expression of vWF, VEGFR1 and R2, VE-cadherin and VCAM-1 when stimulated using medium containing 50ng/ml VEGF and 2\%FBS, after 7days of stimulation. However, there was no expression of PECAM-1. In our study, after 10days of stimulation using EGM+VEGF media we could see an enhanced expression of PECAM-1 indicating a more differentiated phenotype in stimulated cells.

3.2.4. mRNA expression of EC markers by differentiating MSCs-The cells cultured in the EBM showed low mRNA transcript levels for EC marker vWF, VE-Cadherin and PECAM-1. The mRNA transcript levels of vWF (Fig. 6A), PECAM-1 (Fig. 6B) and VE-Cadherin (Fig. 6C) significantly increased in the cells cultured in EGM and EGM +VEGF. There was also a significant increase in VEGF-R1 (Fig. 6D) expression in the cells 
cultured in EGM+VEGF compared to the control. The VEGF-2 receptor mRNA expression increased in cells stimulated with EGM+VEGF (Fig. 6E).

VEGF treatment with EGM resulted in a significant increase in the mRNA expression of VEGF receptors on the differentiating MSCs. The importance of VEGF in vascular development is highlighted by the fact that loss of a single VEGF-A allele results in abnormal vessel development and embryonic death (Ferrara et al., 1996). VEGF signaling is mediated by two receptor tyrosine kinases, called VEGF-R1 and VEGF-R2, respectively. VEGF-R2 plays an important role in hematopoiesis and vasculogenesis, and is the predominant mediator of VEGF-stimulated EC migration, proliferation, survival, and enhanced vascular permeability. Fong et al. (1995) reported that formation of differentiated ECs and their continued proliferation is not affected by the absence of signaling through the VEGF-R1 receptor. But recent reports suggest that VEGF-R1 can augment the VEGF-R2 that can result in increased VEGFR2 phosphorylation (Roskoski, 2008). In the present study, VEGF-R2 might have synergistically acted with VEGF-R1, leading to enhanced phosphorylation events that might have resulted in increased endothelial differentiation. Yamashita et al. (2000) reported that when VEGFR2 ${ }^{+}$cells were cultured on type IV collagen dishes with serum and VEGF165, CD $31^{+}$and VE-cadherin ${ }^{+}$cells were induced, which could form mature vascular like structure and form newly formed vessels. Similar results were observed in the current study where increased VEGF-R2 expression was found, and there was also an increase in the $\mathrm{CD} 31^{+}$and VE-Cadherin ${ }^{+}$cells in the cells cultured in the EGM+VEGF medium. These results indicate that VEGF plays a critical role in maintenance of VEGF receptor expression and differentiation to ECs.

To conclude, the present study demonstrates that porcine MSCs have the potential to differentiate into cells expressing the EC markers in the presence of endothelial growth supplements and VEGF in vitro. The differentiated cells can be of use in generating autologous EC seeded vascular graft in tissue engineering and also in cell therapy for treating cardiovascular diseases.

\section{Acknowledgments}

This work was supported by NIH grants R01HL104516 and R01HL112597, and a grant from the Nebraska Tobacco Settlement Funds (LB692) to Creighton University.

\section{Abbreviations}

Ac-LDL

DiI

EBM

EC

EGM

EGM + VEGF

ESC
Acetylated low density lipoprotein

1,1'-dioctadecyl-3,3,3',3'-tetramethyl-indocarbocyanine perchlorate

Endothelial Basal medium

Endothelial cell

Endothelial Growth medium

Endothelial Growth medium containing 50ng/ml VEGF

Embryonic stem cell

J Tissue Eng Regen Med. Author manuscript; available in PMC 2014 November 01 


$\begin{array}{ll}\text { FBS } & \text { Fetal bovine serum } \\ \text { Flk-1 } & \text { fetal liver kinase 1 } \\ \text { HBSS } & \text { Hank's balanced salt solution } \\ \text { MAPC } & \text { Multipotent adult progenitor cell } \\ \text { PECAM } & \text { Platelet endothelial cell adhesion molecule } \\ \text { TCF/LEF } & \text { T-cell factor/Lymphoid enhancer factor } \\ \text { VE-cadherin } & \text { Vascular endothelial cell cadherin } \\ \text { VEGF } & \text { Vascular endothelial cell growth factor } \\ \text { VEGF -R } & \text { Vascular endothelial cell growth factor receptor } \\ \text { vWF } & \text { von Willebrand factor }\end{array}$

\section{References}

Asahara T, Murohara T, Sullivan A, Silver M, van der Zee R, Li T, Witzenbichler B, Schatteman G, Isner JM. Isolation of putative progenitor endothelial cells for angiogenesis. Science. 1997; 275:964-7. [PubMed: 9020076]

De Val S, Black BL. Transcriptional control of endothelial cell development. Dev Cell. 2009; 16:18095. [PubMed: 19217421]

Dominici M, Le Blanc K, Mueller I, Slaper-Cortenbach I, Marini F, Krause D, Deans R, Keating A, Prockop D, Horwitz E. Minimal criteria for defining multipotent mesenchymal stromal cells. The International Society for Cellular Therapy position statement. Cytotherapy. 2006; 8:315-7. [PubMed: 16923606]

Ferrara N, Carver-Moore K, Chen H, Dowd M, Lu L, O'Shea KS, Powell-Braxton L, Hillan KJ, Moore MW. Heterozygous embryonic lethality induced by targeted inactivation of the VEGF gene. Nature. 1996; 380:439-42. [PubMed: 8602242]

Fong GH, Rossant J, Gertsenstein M, Breitman ML. Role of the Flt-1 receptor tyrosine kinase in regulating the assembly of vascular endothelium. Nature. 1995; 376:66-70. [PubMed: 7596436]

Ghannam S, Bouffi C, Djouad F, Jorgensen C, Noel D. Immunosuppression by mesenchymal stem cells: mechanisms and clinical applications. Stem Cell Res Ther. 2010; 1:2. [PubMed: 20504283]

Jiang Y, Jahagirdar BN, Reinhardt RL, Schwartz RE, Keene CD, Ortiz-Gonzalez XR, Reyes M, Lenvik T, Lund T, Blackstad M, Du J, Aldrich S, Lisberg A, Low WC, Largaespada DA, Verfaillie CM. Pluripotency of mesenchymal stem cells derived from adult marrow. Nature. 2002; 418:41-9. [PubMed: 12077603]

Katoh M, Katoh M. WNT signaling pathway and stem cell signaling network. Clin Cancer Res. 2007; 13:4042-5. [PubMed: 17634527]

Kaufman DS, Lewis RL, Hanson ET, Auerbach R, Plendl J, Thomson JA. Functional endothelial cells derived from rhesus monkey embryonic stem cells. Blood. 2004; 103:1325-32. [PubMed: 14563647]

Kuo TK, Hung SP, Chuang CH, Chen CT, Shih YR, Fang SC, Yang VW, Lee OK. Stem cell therapy for liver disease: parameters governing the success of using bone marrow mesenchymal stem cells. Gastroenterology. 2008; 134:2111-21. 2121 e1-3. [PubMed: 18455168]

Lampugnani MG, Resnati M, Raiteri M, Pigott R, Pisacane A, Houen G, Ruco LP, Dejana E. A novel endothelial-specific membrane protein is a marker of cell-cell contacts. J Cell Biol. 1992; 118:1511-22. [PubMed: 1522121]

Lee OK, Kuo TK, Chen WM, Lee KD, Hsieh SL, Chen TH. Isolation of multipotent mesenchymal stem cells from umbilical cord blood. Blood. 2004; 103:1669-75. [PubMed: 14576065] 
Levenberg S, Golub JS, Amit M, Itskovitz-Eldor J, Langer R. Endothelial cells derived from human embryonic stem cells. Proc Natl Acad Sci U S A. 2002; 99:4391-6. [PubMed: 11917100]

Mannucci PM, Federici AB. Antibodies to von Willebrand factor in von Willebrand disease. Adv Exp Med Biol. 1995; 386:87-92. [PubMed: 8851017]

McCloskey KE, Lyons I, Rao RR, Stice SL, Nerem RM. Purified and proliferating endothelial cells derived and expanded in vitro from embryonic stem cells. Endothelium. 2003; 10:329-36. [PubMed: 14741848]

Nourse MB, Halpin DE, Scatena M, Mortisen DJ, Tulloch NL, Hauch KD, Torok-Storb B, Ratner BD, Pabon L, Murry CE. VEGF induces differentiation of functional endothelium from human embryonic stem cells: implications for tissue engineering. Arterioscler Thromb Vasc Biol. 2010; 30:80-9. [PubMed: 19875721]

Osada M, Ito E, Fermin HA, Vazquez-Cintron E, Venkatesh T, Friedel RH, Pezzano M. The Wnt signaling antagonist Kremen1 is required for development of thymic architecture. Clin Dev Immunol. 2006; 13:299-319. [PubMed: 17162372]

Oswald J, Boxberger S, Jorgensen B, Feldmann S, Ehninger G, Bornhauser M, Werner C. Mesenchymal stem cells can be differentiated into endothelial cells in vitro. Stem Cells. 2004; 22:377-84. [PubMed: 15153614]

Pittenger MF, Mackay AM, Beck SC, Jaiswal RK, Douglas R, Mosca JD, Moorman MA, Simonetti DW, Craig S, Marshak DR. Multilineage potential of adult human mesenchymal stem cells. Science. 1999; 284:143-7. [PubMed: 10102814]

Peister A, Mellad JA, Larson BL, Hall BM, Gibson LF, Prockop DJ. Adult stem cells from bone marrow (MSCs) isolated from different strains of inbred mice vary in surface epitopes, rates of proliferation, and differentiation potential. Blood. 2004; 103:1662-8. [PubMed: 14592819]

Prockop DJ. Marrow stromal cells as stem cells for non-hematopoietic tissues. Science. 1997; 276:714. [PubMed: 9082988]

Reyes M, Dudek A, Jahagirdar B, Koodie L, Marker PH, Verfaillie CM. Origin of endothelial progenitors in human postnatal bone marrow. J Clin Invest. 2002; 109:337-46. [PubMed: 11827993]

Roskoski R Jr. VEGF receptor protein-tyrosine kinases: structure and regulation. Biochem Biophys Res Commun. 2008; 375:287-91. [PubMed: 18680722]

Salven P, Mustjoki S, Alitalo R, Alitalo K, Rafii S. VEGFR-3 and CD133 identify a population of CD34+ lymphatic/vascular endothelial precursor cells. Blood. 2003; 101:168-72. [PubMed: 12393704]

Siekmann AF, Covassin L, Lawson ND. Modulation of VEGF signalling output by the Notch pathway. Bioessays. 2008; 30:303-13. [PubMed: 18348190]

Toma C, Pittenger MF, Cahill KS, Byrne BJ, Kessler PD. Human mesenchymal stem cells differentiate to a cardiomyocyte phenotype in the adult murine heart. Circulation. 2002; 105:93-8. [PubMed: 11772882]

van Amerongen R, Nusse R. Towards an integrated view of Wnt signaling in development. Development. 2009; 136:3205-14. [PubMed: 19736321]

Voyta JC, Via DP, Butterfield CE, Zetter BR. Identification and isolation of endothelial cells based on their increased uptake of acetylated-low density lipoprotein. J Cell Biol. 1984; 99:2034-40. [PubMed: 6501412]

Wakitani S, Saito T, Caplan AI. Myogenic cells derived from rat bone marrow mesenchymal stem cells exposed to 5-azacytidine. Muscle Nerve. 1995; 18:1417-26. [PubMed: 7477065]

Xu J, Liu X, Jiang Y, Chu L, Hao H, Liua Z, Verfaillie C, Zweier J, Gupta K, Liu Z. MAPK/ERK signalling mediates VEGF-induced bone marrow stem cell differentiation into endothelial cell. $\mathrm{J}$ Cell Mol Med. 2008; 12:2395-406. [PubMed: 18266967]

Yamashita J, Itoh H, Hirashima M, Ogawa M, Nishikawa S, Yurugi T, Naito M, Nakao K, Nishikawa S. Flk1-positive cells derived from embryonic stem cells serve as vascular progenitors. Nature. 2000; 408:92-6. [PubMed: 11081514]

Zuk PA, Zhu M, Mizuno H, Huang J, Futrell JW, Katz AJ, Benhaim P, Lorenz HP, Hedrick MH. Multilineage cells from human adipose tissue: implications for cell-based therapies. Tissue Eng. 2001; 7:211-28. [PubMed: 11304456] 

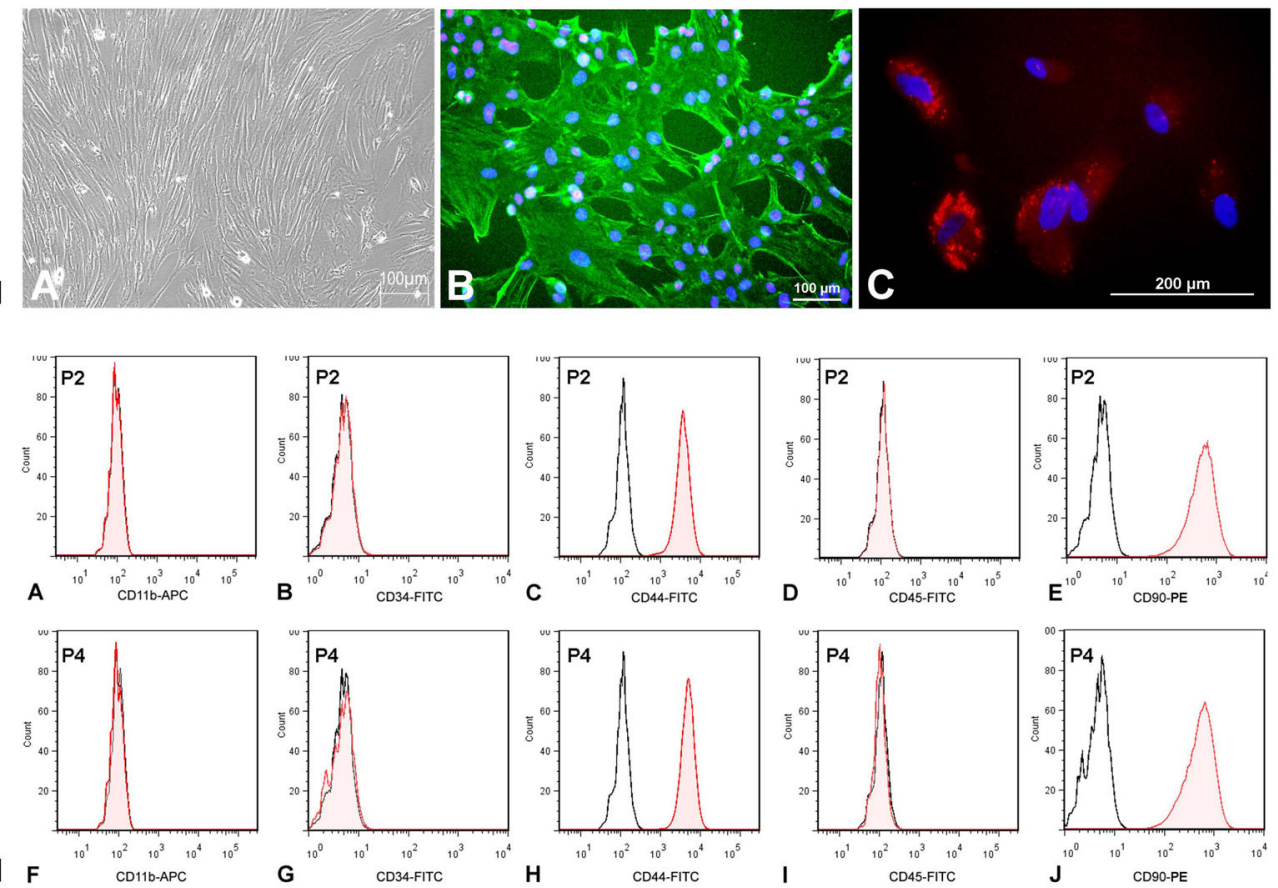

Figure 1. MSC characterization

I. (A) MSCs showing fibroblastoid morphology in culture (B) Immunostaining of MSCs showing vimentin (green) and C) CD105 (red) staining. Cell nuclei were stained with DAPI.

II. Immunophenotyping of MSCs in second (P2) and fourth (P4) passages of culture. Flow cytometry data showing absence of CD11b (A), CD34 (B), CD45 (D), and presence of CD44 (C) and CD90 (E) (MSC markers). The unshaded peak shows the profile of the isotype control. Flow cytometry was done on FACS Aria Flow Cytometry System (BD Biosciences, USA). FITC, APC and PE-labeled IgG (BD Pharmingen, USA) served as isotype controls. 


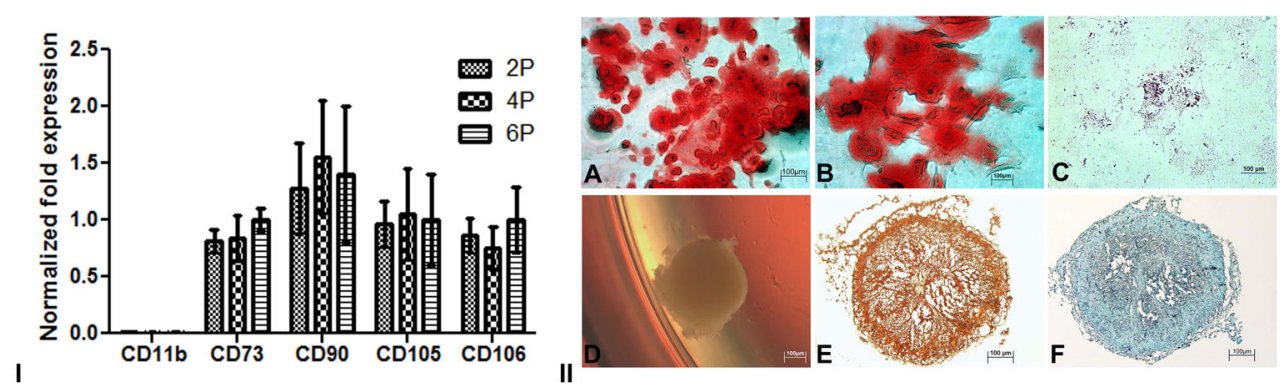

Figure 2. MSC characterization

I. mRNA expression of MSC markers. Real Time PCR graph showing negative expression of CD11b and positive expression of CD73, CD90, CD105 and CD106 in second, fourth and sixth passages in isolated MSCs. GAPDH was used as the house keeping gene. The specificity of the primers was analyzed by running a melt curve. II. Multilineage differentiation of MSCs in vitro. A) Alizarin S red staining of MSC culture after 14 days stimulation with osteogenic differentiation medium (DM) B) High magnification image showing osteogenic differentiation C) Oil O Red staining after 21 days of stimulation with adipogenic DM; D) Chondrogenic pellet formed after 21 days of stimulation with chondrogenic DM, E) Safranin O staining of cryosections of chondrogenic pellet, F) Alcian blue staining of cryosections of chondrogenic pellet. 


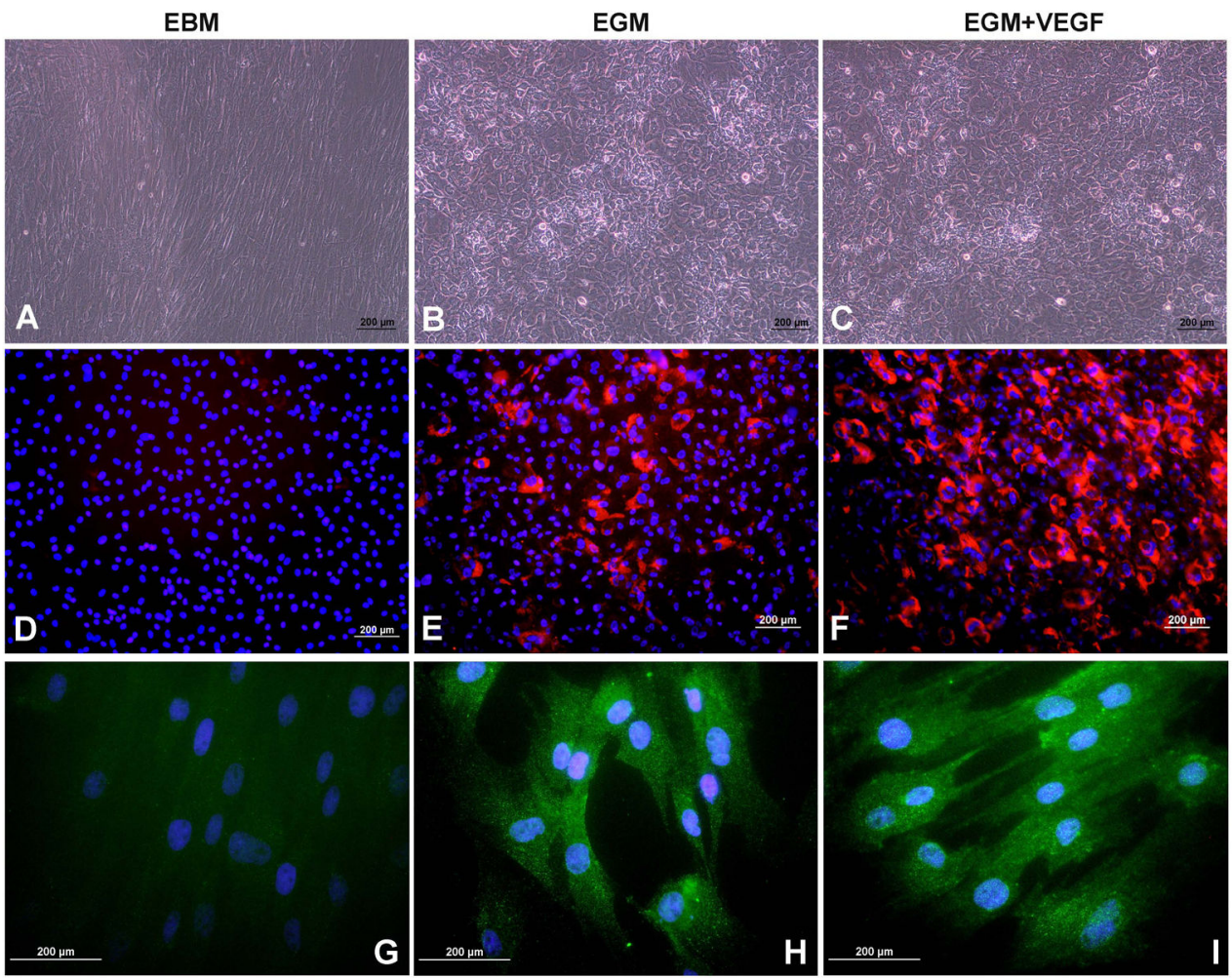

Figure 3. EC differentiation of MSCs after 10days of stimulation

Morphology of cells after 10 days of differentiation in A) EBM, B) EGM and C) EGM + VEGF medium. DiI-labeled-Acetylated LDL uptake in cells induced by D) EBM, E) EGM and F) EGM + VEGF medium. vWF staining of cells in G) EBM, H) EGM and I) EGM + VEGF medium 


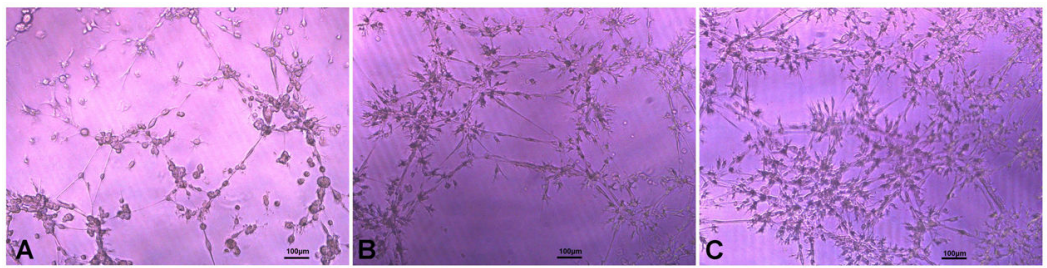

Figure 4. In vitro angiogenesis assay

In vitro tubule formation of cells induced in (A) EBM, (B) EGM and (C) EGM + VEGF seeded on ECM gel after 12h of seeding. 

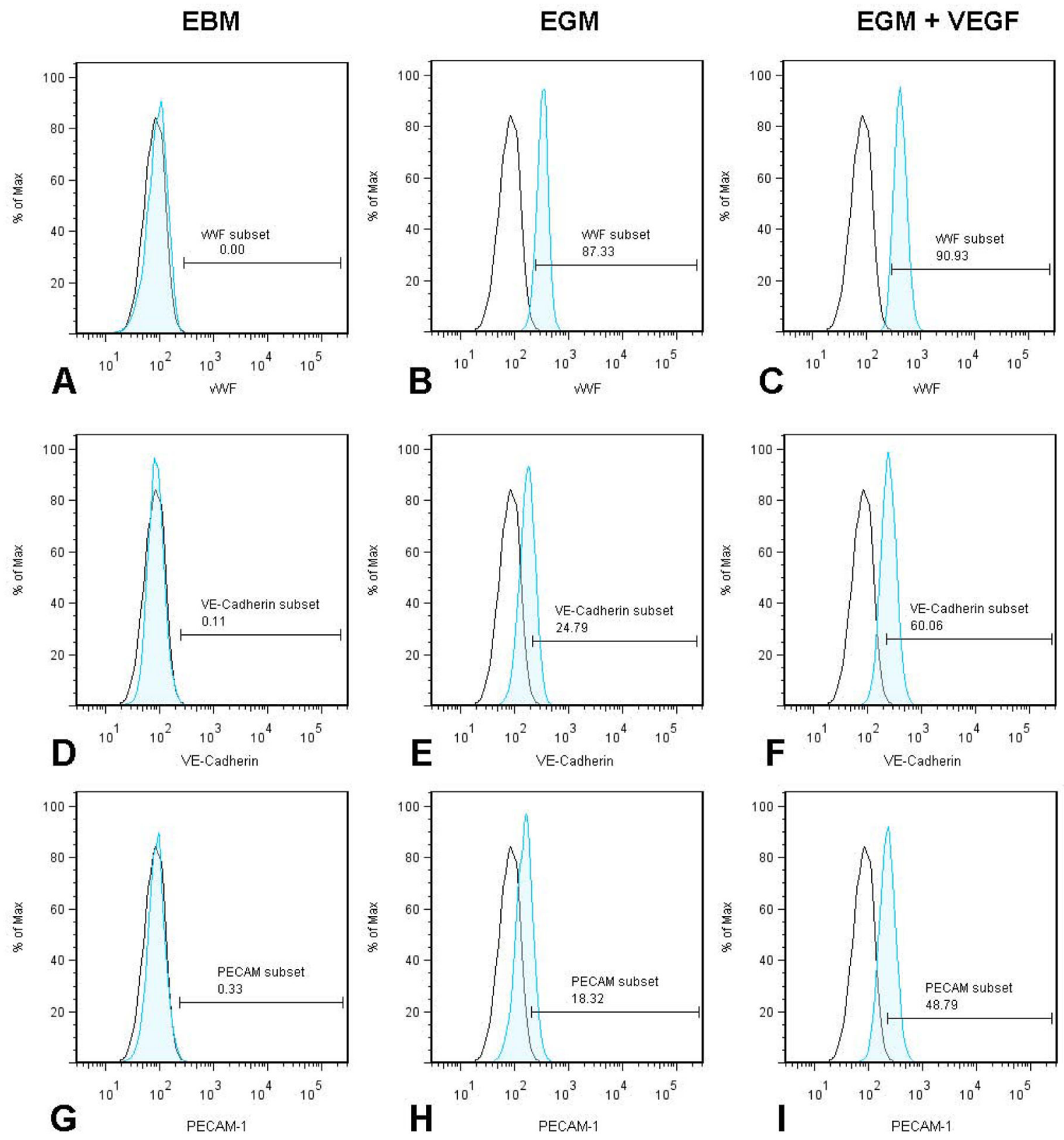

Figure 5. Immunophenotyping of differentiated cells for EC markers

Panel 1 represents the cells stimulated in EBM media for vWF (A), VE-Cadherin (D) and PECAM-1 (G). Panel 2 represents the cells stimulated in EGM media for vWF (B), VECadherin (E) and PECAM-1 (H). Panel 3 represents the cells stimulated in EGM + VEGF media for vWF (C), VE-Cadherin (F) and PECAM-1 (I). The unshaded peak shows the profile of the isotype control. 

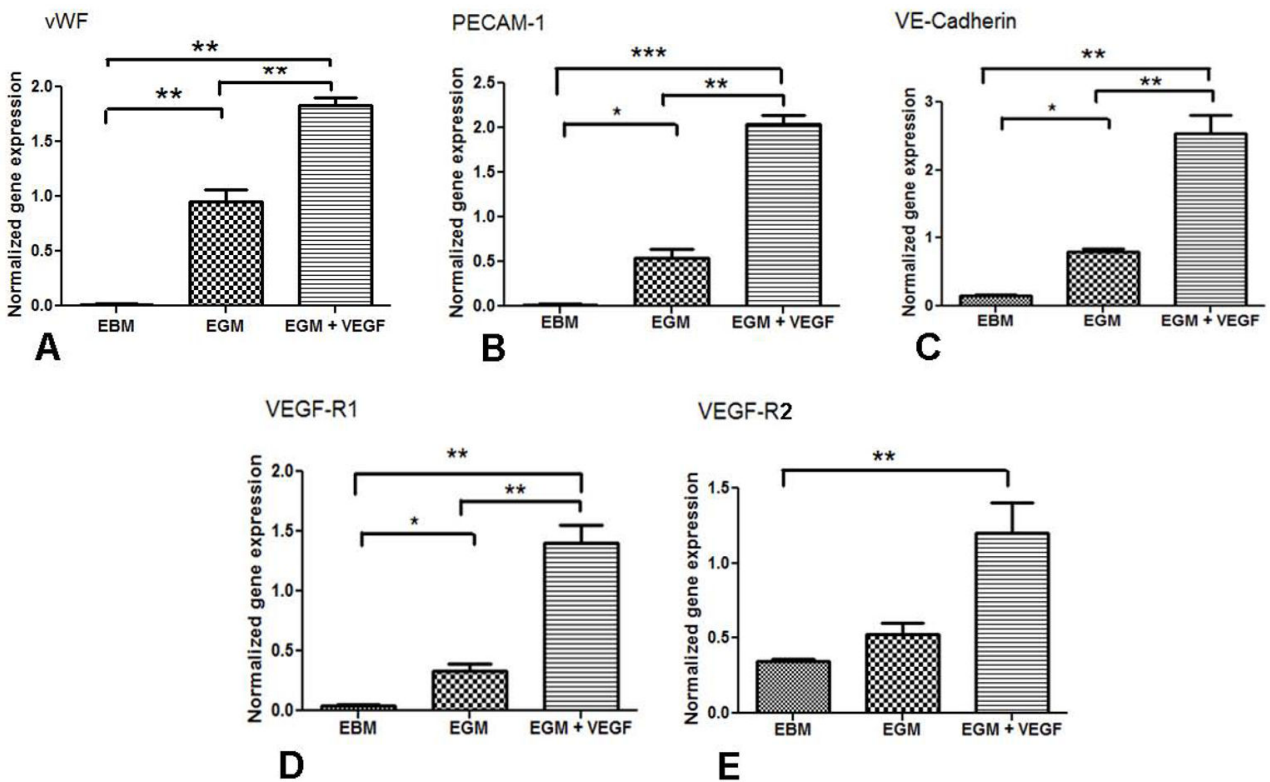

Figure 6. mRNA expression of EC markers in differentiated cells Quantitative PCR data showing expression levels of (A) vWF, (B) PECAM-1, (C)VECadherin, (D) VEGF-R1 and (E) VEGF-R2 in cells cultured in EBM, EGM and EGM + VEGF for 10days. $(* * * p<0.001, * * p<0.01$ and $* p<0.05)$. 
Table 1

Primers for Real Time PCR

\begin{tabular}{|l|l|l|l|}
\hline Gene & Reference sequence & Primers & Product length \\
\hline CD11b & NM_000632.3 & $\begin{array}{l}\text { FP: 5'-CAGCTGCTGGCCTGTGGTCC-3' } \\
\text { RP: 5'-GGGCTGCTGCCGTAGGTTGG-3' }\end{array}$ & 102 \\
\hline CD73 & NM_002526.2 & $\begin{array}{l}\text { FP: 5'-GCAAGTGCGTCAACGCCAGC-3' } \\
\text { RP: 5'-ATGGCATCGTAGCGCAGGGC-3' }\end{array}$ & 193 \\
\hline CD90 & NM_001146129.1 & $\begin{array}{l}\text { FP: 5'-ACAGTCTTGCAGGTGGCCCG-3' } \\
\text { RP: 5'-TCAGGGACCCCCACAGTGCC-3' }\end{array}$ & 179 \\
\hline CD105 & NM_000118.2 & $\begin{array}{l}\text { FP: 5'-CGCTCCCTCTGGCTGTTGCC-3' } \\
\text { RP: 5'-TGTCACCTCGCCCCTCTCGG-3' }\end{array}$ & 116 \\
\hline CD106 & NM_001078.2 & $\begin{array}{l}\text { FP: 5'-CCTGGACCCCGGATTGCTGC-3' } \\
\text { RP: 5'-ACAGGGCTCAGGGTCAGCGT-3' }\end{array}$ & 164 \\
\hline vWF & NM_000552.3 & $\begin{array}{l}\text { FP: 5'-GCCTGTGGGAGCAGTGCCAG-3' } \\
\text { RP: 5'-GGGCGTACTCCAGGAGGGCA-3' }\end{array}$ & 159 \\
\hline PECAM 1 & NM_000442.3 & $\begin{array}{l}\text { FP: 5'-GGGGCCACGATGTGGCTTGG-3' } \\
\text { RP:5'-CGCGAAGCACTGCAGGGTCA-3' }\end{array}$ & 156 \\
\hline VE-Cadherin & NM_001795.3 & $\begin{array}{l}\text { FP: 5'-GTGCAACGAGCAGGGCGAGT-3' } \\
\text { RP:5'-CTCGTGGATCTCCGGCACGC -3' }\end{array}$ & 190 \\
\hline VEGF-R1 & NM_001159920.1 & $\begin{array}{l}\text { FP: 5'-GGCCCCAGGGGTGCAAGATG-3' } \\
\text { RP:5'-TCGCATGAGAGGAGGGAGGGGA-3' }\end{array}$ & 109 \\
\hline VEGF-R2 & NM_002253.2 & $\begin{array}{l}\text { FP: 5'-CTTGCGCGCCGCAGAAAGTC-3' } \\
\text { RP: 5'-CCGCAGCGCAGGACAGTTGA-3' }\end{array}$ & 129 \\
\hline
\end{tabular}

